\title{
A Case of Pseudoangiomatous Stromal Hyperplasia of the Breast Presenting with Chest Pain
}

\author{
Misato Amenomori ${ }^{1}$, Tomayoshi Hayashi ${ }^{*}, 2$, Kuniko Abe ${ }^{2,3}$, Noriaki Itoyanagi ${ }^{4}$ and Shigeru Kohno \\ ${ }^{I}$ Second Department of Internal Medicine, Nagasaki University School of Medicine, Nagasaki, Japan \\ ${ }^{2}$ Department of Pathology, Nagasaki University Hospital, Nagasaki, Japan \\ ${ }^{3}$ Division of Pathology, Nagasaki University School of Medicine, Nagasaki, Japan \\ ${ }^{4}$ Itoyanagi Breast Clinic, Nagasaki, Japan
}

\begin{abstract}
Pseudoangiomatous stromal hyperplasia is a rare, benign, breast disease characterized by dense, collagenous proliferation of mammary stroma, forming interanastomosing capillary-like spaces. Previously reported cases presented with a palpable breast mass or continuous breast enlargement, or were unexpectedly detected on mammography or ultrasonography. However, cases presenting only with pain as the initial symptom have not been reported. In this report, we describe a case of pseudoangiomatous stromal hyperplasia that presented with intermittent dull chest pain. A $2.6 \times 1.1 \mathrm{~cm}$, well-defined, hypoechoic mass was noted in the breast on ultrasonography, and an excisional biopsy was performed. On pathology, the tumor showed stromal hyperplasia and vessel-like slit structures. Immunohistochemical staining for vimentin, CD34, CD31, and Factor VIII-related antigen was compatible with pseudoangiomatous stromal hyperplasia. The dull chest pain disappeared after the excision. Pseudoangiomatous stromal hyperplasia is often described as painless. However, chest pain could also be an initial symptom.
\end{abstract}

Keywords: Pseudoangiomatous stromal hyperplasia (PASH), chest pain, benign breast tumor.

\section{INTRODUCTION}

Pseudoangiomatous stromal hyperplasia (PASH), first reported by Vuitch et al., is a benign proliferative disease of the breast [1]. PASH is characterized by dense, collagenous proliferation of mammary stroma, forming interanastomosing capillary-like spaces $[2,3]$. Some patients present with single or multiple, rubbery soft masses without pain or with a few months' history of breast enlargement $[4,5]$. In other patients, the tumors are first detected on mammography or ultrasonography [6]. In this report, a rare case of PASH that presented with intermittent dull chest pain as the initial symptom is described.

\section{CASE REPORT}

A 30-year-old woman noticed dull pain in the left lateral chest, just below the lower portion of the breast. She was pre-menopausal and had no history of oral contraceptive use or hormone replacement therapy. The pain was intermittent and sometimes increased when breathing deeply. After 6 months, she detected an elastic-hard nodule in her left breast and visited the clinic. Physical examination revealed a firm, elastic-hard, well-defined, easily movable, $3.0-\mathrm{cm}$ nodule in the lower half of the left breast. Mammography revealed no discrete mass or calcification (category 1). Ultrasonography of the left breast showed a $26 \times 11 \mathrm{~mm}$, well-defined,

\footnotetext{
*Address correspondence to this author at the Department of Pathology, Nagasaki University Hospital, Sakamoto, Nagasaki 852-8501, Japan; Tel: +81 95819 7562; Fax: +81 95819 7564; E-mail: toma@nagasaki-u.ac.jp
}

homogenously hypoechoic mass with small cysts, 5 to $6 \mathrm{~mm}$ in diameter (Fig. 1). A similar hypoechoic mass of $7.8 \times 2.9$ $\mathrm{mm}$ was also seen in the right breast. Fine needle aspiration cytology from the larger nodule in the left breast showed some clusters of epithelial cells with small papillary structures and myoepithelial cells, which strongly suggested fibroadenoma.

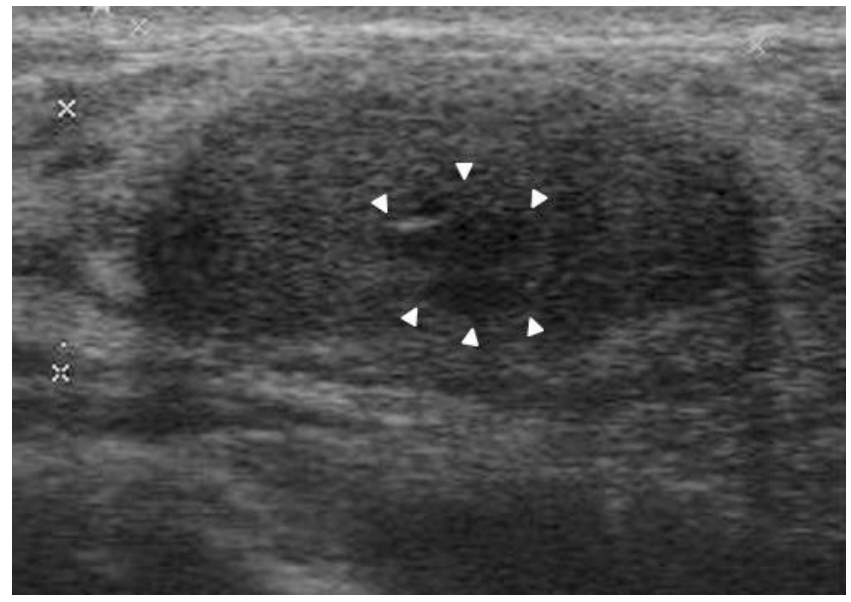

Fig. (1). Ultrasonography shows a $26 \times 11 \mathrm{~mm}$, well-defined, homogenously hypoechoic mass in the lower half of the left breast. Small cysts, 5 to $6 \mathrm{~mm}$ in diameter, are seen in the mass (arrows heads).

An excisional biopsy of the larger nodule was performed at the patient's request. The excised tumor was well demarcated and had a smooth external surface. Gross examination revealed a $30 \times 25 \times 15 \mathrm{~mm}$, rubbery-hard mass (Fig. 2a). 


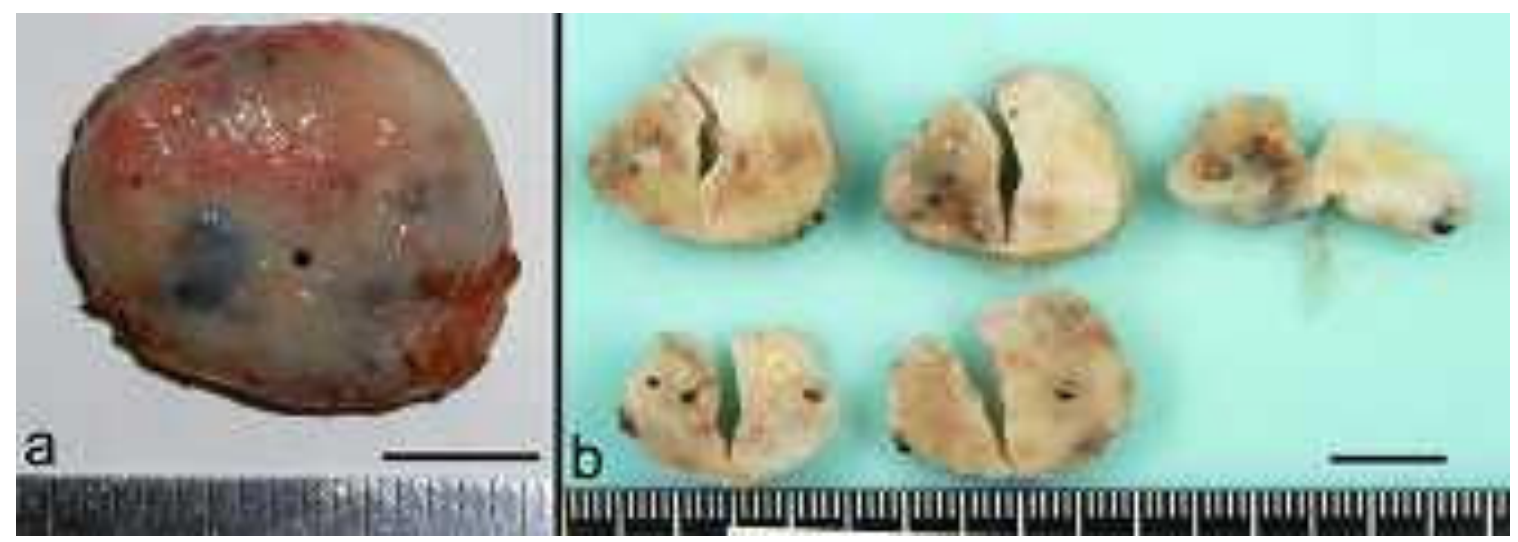

Fig. (2). (a) The cut surface shows a homogenous, grayish white appearance with small cysts filled with dark red fluid. (b) Gross appearance of the $30 \times 25 \times 15 \mathrm{~mm}$ rubbery-hard mass shows a well demarcated, smooth external surface. Scale bar $=10 \mathrm{~mm}$.

The cut surface of the tumor was homogenous, fibrous, and white in color (Fig. 2b). There were small cysts filled with dark red fluid, and no hemorrhage or necrosis was present.

On histological examination, the tumor was predominantly composed of markedly increased fibrous stroma (Fig. 3a, b). The tumor did not contain adipose tissue, and there were no pathologic features of fibroadenoma or phyllodes tumor. The mass was well circumscribed but not encapsulated. The fibrous stroma contained numerous slit-like structures. The slits were covered by spindle cells, and the nuclei of the spindle cells appeared bland and lacked atypia or mitotic activity.
Immunohistochemically, the spindle cells forming the slit-like spaces were negative for CD31, Factor VIII-related antigen (Fig. 3c), and D2-40, suggesting that they were neither capillaries nor lymph vessels. The spindle cells were positive for CD34 and vimentin (Fig. 3d), and negative for smooth-muscle-actin. These pathological features are characteristic of pseudoangiomatous stromal hyperplasia (PASH) $[2,3]$. The nuclei of the spindle cells covering the slit-like spaces were negative for progesterone (Fig. 4a) and estrogen (Fig. 4b) receptors. There were no nerve fibers within the tumor. The antibodies used in this case are listed in the Table 1.

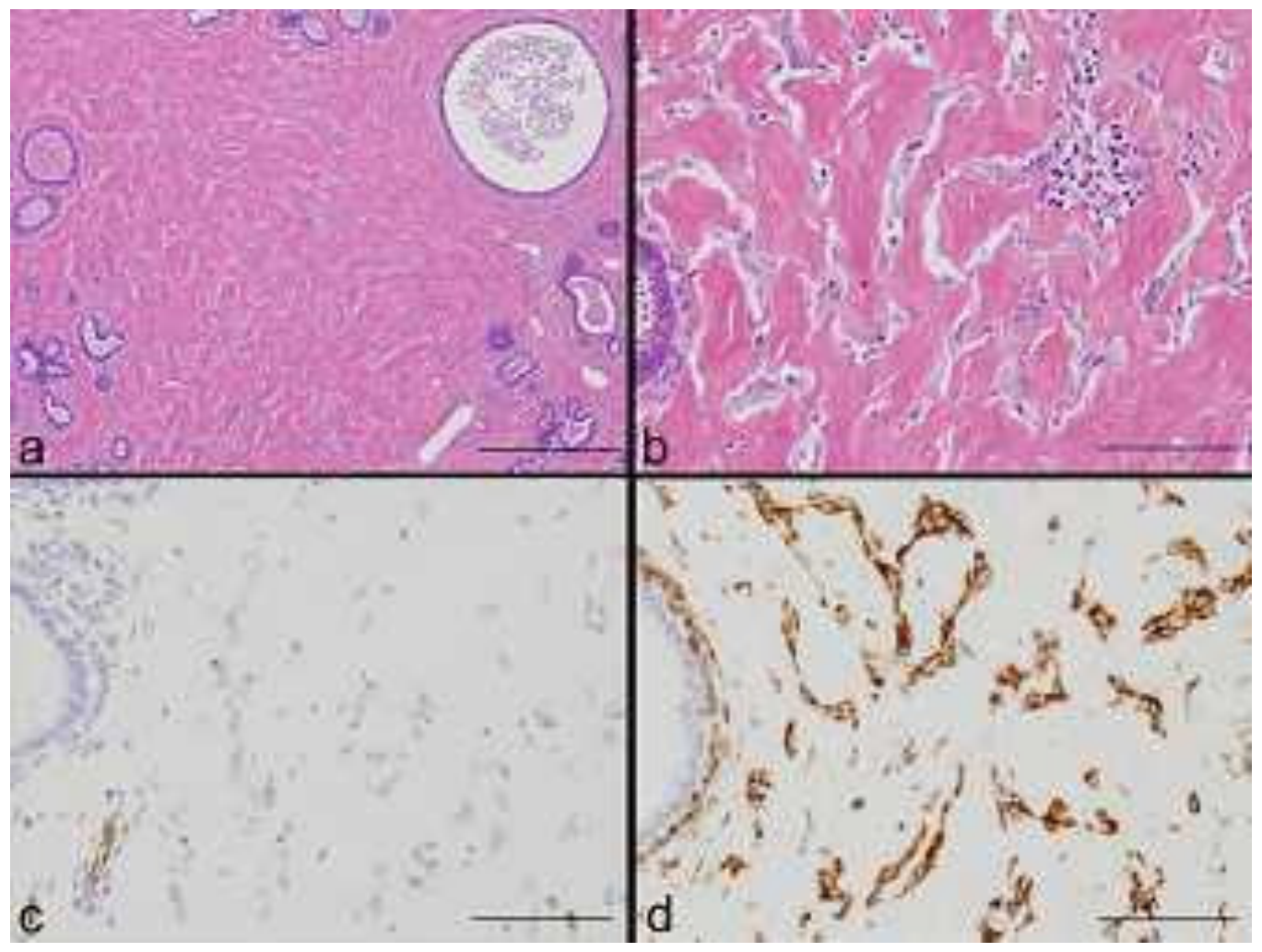

Fig. (3). Histological examination shows densely collagenous stroma with numerous slit-like structures. (a, b) Hematoxylin-eosin stain (scale bar: $\mathbf{a}=500 \mu \mathrm{m}, \mathbf{b}=100 \mu \mathrm{m}$ ). (c): Immunohistochemical staining for Factor VIII-related antigen, shows a negative reaction in the spindle cells covering the slit-like spaces, in contrast to the positive reaction in the vascular endothelial cells $(\mathrm{scale} b a r=100 \mu \mathrm{m}) .(\mathbf{d})$ : Immunohistochemical staining for vimentin shows a positive reaction in the spindle cells (scale bar $=100 \mu \mathrm{m})$. 


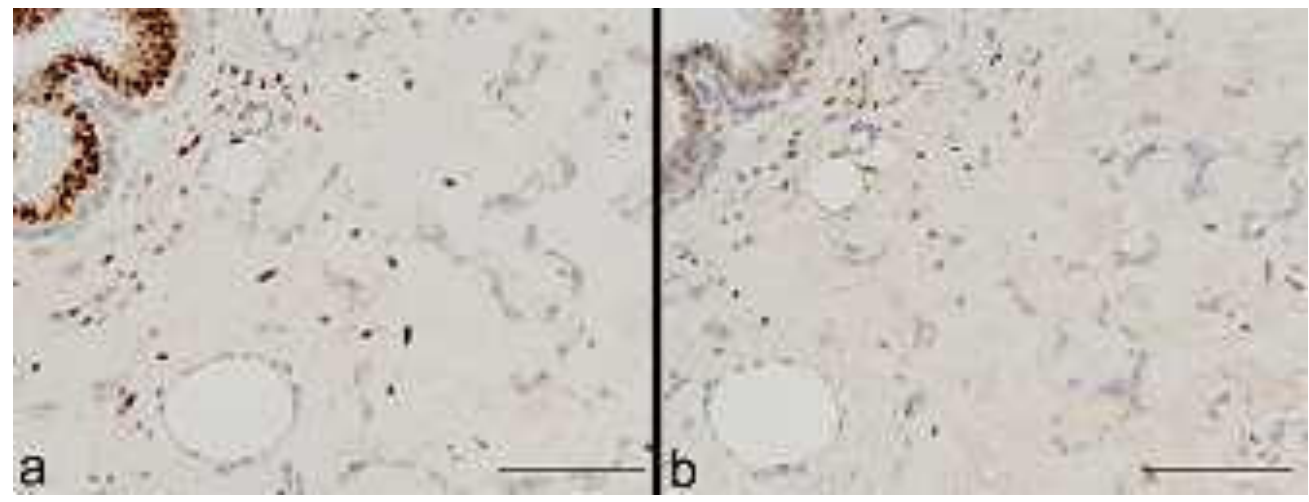

Fig. (4). (a) Immunohistochemical staining for progesterone receptor shows no reaction in the nuclei of the spindle cells covering the slit-like spaces. A slight positive reaction is seen in some stromal cells. The nuclei of the epithelial cells of the mammary ducts are strongly positive (scale bar $=100 \mu \mathrm{m}$ ). (b) Immunohistochemical staining for estrogen receptor shows mostly a negative reaction in the nuclei of the spindle cells covering the slit-like spaces. Positive reaction is seen in the nuclei of the epithelial cells of the mammary ducts $(\mathrm{scale}$ bar $=100 \mu \mathrm{m})$.

Soon after the excision, the chest pain disappeared. At present, 12 months after the operation, there has been no evidence of local recurrence or enlargement of the residual nodule in the right breast.

\section{DISCUSSION}

PASH is a benign proliferative disease of the breast stroma, which was first reported by Vuitch et al. [1]. Although it does not actually constitute vasoformative proliferation, the histological pattern mimics angiosarcoma, and this benign stromal lesion tends to be misdiagnosed as low grade angiosarcoma. Therefore, the term pseudoangiomatous was proposed [2].

In most cases, PASH is first noticed as single or multiple, palpable breast masses $[4,7]$ or as continuous breast enlargement [5]. Some reported cases were asymptomatic and detected unexpectedly on mammography or ultrasonography [6]. Although the lesions are generally described as painless $[2,4]$, one case presented with mild pain and peau d'orange change of the overlying skin, which clinically resembled inflammatory breast carcinoma or mastitis [8].

The pain of the breast is subcategorized to that which originates in the mammary gland and that due to other causes [9]. Most mammary gland pain is caused by mastopathy, while other possibilities include acute or chronic mastitis, mastodynia, mammary duct ectasia, and breast cancer. Dis- eases that are associated with the mammary gland but may cause breast pain are Mondor disease, Tietz syndrome, intercostal nerve disorders, or rib fractures. If the pain is on the left side, cardiac diseases, such as angina pectoris, could also be responsible [9]. In the present case, the patient first noticed pain in the chest, just below the lower portion of the left breast. The pain did not show an apparent correlation with the menstrual cycle or inflammatory changes in the breast. As for the mechanism of the pain, involvement of the nerves within the lesion, or stimulation or compression of neighboring nerves may have been responsible. In the present case, there were no nerves within the tumor. Therefore, the pain was probably caused by stimulation of peripheral nerves surrounding the tumor, or nerves located slightly more distant from the tumor, for example, intercostal nerves or pectoral nerves.

On imaging examinations, PASH has no specific features that contribute to making the diagnosis $[6,10]$. Mammograms reveal a round or orbital, circumscribed or partially circumscribed, non-calcified mass. On ultrasonography, a homogenously hypoechoic, solid mass is common, with a variety of iso- and high-echoic cases, or there may be small internal cysts $[6,10,11]$.

Microscopically, the tumors are composed of intermixed stromal and epithelial elements, and the lobular and duct structures of the breast parenchyma are separated by an increased amount of fibrous stroma. In the stromal area, ves-

Table. 1. List of the Antibodies Used for the Immunohistochemical Staining

\begin{tabular}{|c|c|c|}
\hline Antibody & Dilution & Company, City \\
\hline \hline CD31 (M0823) & $1: 50$ & Dako Japan, Tokyo, Japan \\
\hline CD34 (\#413111) & ready to use & Nichirei Biosciences Inc., Tokyo, Japan \\
\hline D2-40 (M3619) & $1: 50$ & Dako Japan, Tokyo, Japan \\
\hline Estrogen receptor (M7047) & $1: 50$ & Dako Japan, Tokyo, Japan \\
\hline Factor VIII-related antigen (A0082) & $1: 400$ & Dako Japan, Tokyo, Japan \\
\hline Progesterone receptor (M3569) & $1: 50$ & Dako Japan, Tokyo, Japan \\
\hline Smooth-muscle-actin (M0851) & $1: 100$ & Dako Japan, Tokyo, Japan \\
\hline Vimentin (M7020) & $1: 200$ & Dako Japan, Tokyo, Japan \\
\hline
\end{tabular}


sel-like slits surrounded by spindle cells are seen randomly, and on immunohistochemistry, the spindle cells are positive for vimentin and CD34, and negative for Factor VIII-related antigen and cytokeratin $[1,4,12,13]$. The morphological spectrum of cellular proliferation and staining qualities indicates that myofibroblasts play a major role in the histogenesis of PASH [7, 13]. Electron microscopy also shows the incomplete fibroblastic nature of the tumor $[1,8]$.

In $\mathrm{PASH}$, pleomorphism and hyperchromasia of the stroma cell nuclei are minimal to absent, and the slit-like spaces only very rarely contain red blood cells; these histologic features are important to distinguish it from low grade angiosarcoma [1].

Wide local excision is recommended as the standard treatment of PASH [1]. Incomplete excision may result in local recurrence, with the need for mastectomy [5]. On the other hand, a case with multiple lesions may disappear spontaneously [7]. Therefore, some authors recommend conservative management with periodic follow-up [14]. As for systemic treatment, tamoxifen is usually suggested [15], though it is not always effective [16]. In the present case, the patient preferred earlier excision, since it can instantly lead to the proper diagnosis with a smaller surgical scar.

A hormonal etiology is likely for PASH, though it is still controversial. That is, hyperplasia of the mammary stroma cells is induced by an excessive response to progesterone [1, 17], and positive immunoreactivity for progesterone receptors, often intensely positive in stroma cells, is considered to support this process [18]. However, in the present case, the nuclei of stromal cells were negative for progesterone receptors, which does not support a hormonal etiology for PASH.

\section{CONCLUSION}

A case of PASH that presented with intermittent dull chest pain was described. PASH is often described as painless. However, chest pain could also be an initial symptom.

\section{REFERENCES}

[1] Vuitch MF, Rosen PP, Erlandson RA. Pseudoangiomatous hyperplasia of mammary stroma. Hum Pathol 1986; 17: 185-91.

[2] Rosen PP, Oberman HA. Tumors of the mammary gland. In: Atlas of Tumor Pathology. Washington, D.C., USA: Armed Forces Institute of Pathology 1992; pp. 303-13.
[3] In: Tavassoli FA, Devilee P, Eds. Pathology and genetics of tumours of the breast and female genital organs. Lyon: International Agency for Research on Cancer 2003.

[4] Taira N, Ohsumi S, Aogi K, et al. Nodular pseudoangiomatous stromal hyperplasia of mammary stroma in a case showing rapid tumor growth. Breast Cancer 2005; 12: 331-6.

[5] Singh KA, Lewis MM, Runge RL, Carlson GW. Pseudoangiomatous stromal hyperplasia: a case for bilateral mastectomy in a 12year-old girl. Breast J 2007; 13: 603-6.

[6] Polger MR, Denison CM, Lester S, Meyer JE. Pseudoangiomatous stromal hyperplasia: mammographic and sonographic appearances. AJR Am J Roentgenol 1996; 166: 349-52.

[7] Okoshi K, Ogawa H, Suwa H, Saiga T, Kobayashi H. A case of nodular pseudoangiomatous stromal hyperplasia (PASH). Breast Cancer 2006; 13: 349-53.

[8] Ibrahim RE, Sciotto CG, Weidner N. Pseudoangiomatous hyperplasia of mammary stroma. Some observations regarding its clinicopathologic spectrum. Cancer 1989; 63: 1154-60.

[9] In: Izuo M, Senoo T, Eds. Mastopathy: diseases of the Breast. First Eds. Tokyo, JAPAN: Kanehara \& Co. Ltd; 1986; p. 147. (reference in Japanese)

[10] Cohen MA, Morris EA, Rosen PP, Dershaw DD, Liberman L, Abramson AF. Pseudoangiomatous stromal hyperplasia: mammographic, sonographic, and clinical patterns. Radiology 1996; 198: 117-20.

[11] Mercado CL, Naidrich SA, Hamele-Bena D, Fineberg SA, Buchbinder SS. Pseudoangiomatous stromal hyperplasia of the breast: sonographic features with histopathologic correlation. Breast J 2004; 10: 427-32.

[12] Milanezi MF, Saggioro FP, Zanati SG, Bazan R, Schmitt FC. Pseudoangiomatous hyperplasia of mammary stroma associated with gynaecomastia. J Clin Pathol 1998; 51: 204-6.

[13] Powell CM, Cranor ML, Rosen PP. Pseudoangiomatous stromal hyperplasia (PASH): a mammary stromal tumor with myofibroblastic differentiation. Am J Surg Pathol 1995; 19: 270-7.

[14] Salvador R, Lirola JL, Dominguez R, Lopez M, Risueno N. Pseudo-angiomatous stromal hyperplasia presenting as a breast mass: imaging findings in three patients. Breast 2004; 13: 431-5.

[15] Pruthi S, Reynolds C, Johnson RE, Gisvold JJ. Tamoxifen in the management of pseudoangiomatous stromal hyperplasia. Breast $\mathrm{J}$ 2001; 7: 434-9.

[16] Seltzer MH, Kintiroglou M. Pseudoangiomatous hyperplasia and response to tamoxifen therapy. Breast J 2003; 9: 344 .

[17] Anderson C, Ricci A, Jr., Pedersen CA, Cartun RW. Immunocytochemical analysis of estrogen and progesterone receptors in benign stromal lesions of the breast: evidence for hormonal etiology in pseudoangiomatous hyperplasia of mammary stroma. Am J Surg Pathol 1991; 15: 145-9.

[18] Tavassoli FA. Pseudoangiomatous Stromal Hyperplasia. In: Pathology of the Breast. second Eds. McGraw-Hill Professional Publishing 1999; pp. 663-7. 